\title{
Free Vibration of a CLD Combination Cylindrical Shells
} Hongjun Ren ${ }^{1 a}$, Boping Wang ${ }^{2 b}$, Xuyuan Song ${ }^{3 c}$, Qingkai Han* ${ }^{4 d}$

${ }^{13}$ School of Mechanical Engineering, Northeastern University, Shenyang, 110819, China

${ }^{2}$ Department of Flight Theory, Aviation University of Air Force, Changchun, 130022, China.

a rhj781226@163.com, ${ }^{\mathrm{b}}$ wbp04620@sina.com, ${ }^{* d}$ qhan@mail.neu.edu.cn (Corresponding author)

Keywords: Love shell theory, CLD cylindrical shell, transfer matrix, dissipation factor

\begin{abstract}
Based on elasticity plate theory and the theory of viscoelasticity, the solution of the natural frequency and dissipation factor are given under different boundary conditions. In this paper, the differential equation of the cylindrical shells is set up based on Love shell theory. Then the transfer matrix method is introduced to calculate the entirety transfer matrix and the expression of different frequency and dissipation factor. At last the natural character of CLD cylindrical shell under two different boundary conditions is researched.
\end{abstract}

\section{Introduction}

The composite laminated shell-plate combination is a classic structure in modern industry. Compared with traditional cylindrical shell, the combination cylindrical shell is much lighter, higher stress and more design freedom. Constrained Layer Damping (CLD) is a typical composite laminated structure thus it is necessary to research the vibration character of CLD[1,2]. Current combination cylindrical shells is composition by damping layer and restraint layer and both of them are placed inside the elastic layer. Most of the cylindrical shells, operating under complex condition, treat the outer side as working surface in this case the constrained layer damping cannot be placed on the working surface.

Current methods for the natural vibration analysis of the combination cylindrical shells can be classified into two categories. Enqi Li and Yongjun Lei et al. [3] based on Hamilton principle developed a transfer matrix method and analyzed the dynamic character of CLD beam and CLD shell. Then they derived the first-order ordinary differential matrix equation whose state vector are displacement and higher order derivative. Yu Xiang and Yuying Huang et al. [4] based on the basic function of cylindrical shells, considering the effect of shear energy consumption of viscoelastic layer, and they derived the integration of ordinary differential equations and analyzed the vibration and damp character of the cylindrical shells.

Considering the fact that analyses in this paper are focused on vibration character of CLD shell, thus a transfer matrix method is researched. The state equation is set up based on the differential equations. The displacement and force are treated as state variable, forming a transfer matrix. Then the expression of natural frequency and dissipation factor is calculated under different boundary conditions. Finally, a group of solution of CLD shell is given in this document.

\section{Theoretical formulation}

Considering a model of cylindrical shell shown in Fig 1. L, R and $H$ are the length, radius and thickness of the shell. The cylindrical coordinate system is introduced to this paper, where the displacement $u, v$ and $w$ express the shifting of $\mathrm{x}-,, \mathrm{y}-$ and $\mathrm{z}$-axis. The shell is divided into $N$ 
section and the length of each part is $L_{1}, L_{2} \cdots L_{k-1}, L_{k}(k \leq N)$. Define a coordinate system which is determined by the orthogonal curvilinear of generatrix and parallel of the shell. Selecting a point of the middle surface and assuming the point as $(x, \theta), x$ is the length, $\theta$ is the subtended angle which is a constant.
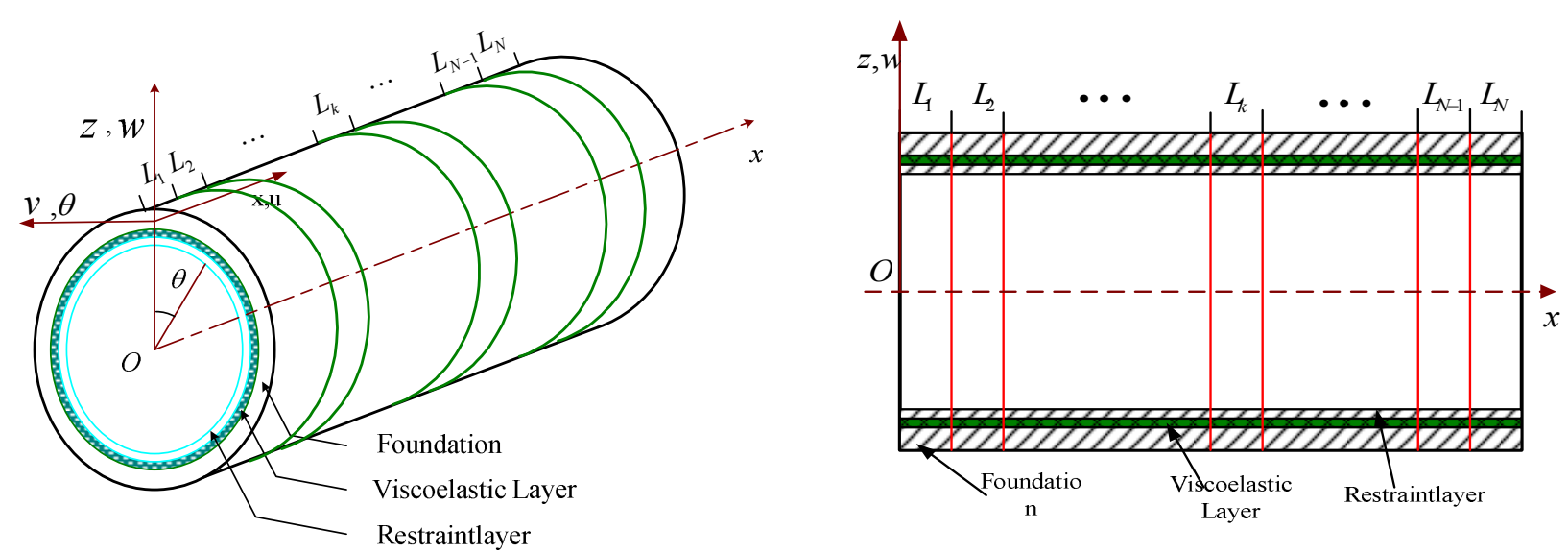

Fig. 1 Cylindrical shell model separated in axial direction

Based on the LOVE theory and considering the equivalent Kirchhoff plane shear $V_{x}$ and transverse shear $S_{x}[5,6]$.

$$
\begin{aligned}
& V_{x}=Q_{x}+\frac{1}{R} \frac{\partial M_{x \theta}}{\partial \theta} \\
& S_{x}=N_{x \theta}+\frac{1}{R} M_{x \theta}
\end{aligned}
$$

The equations of vibration for a cylindrical shell can be written as follow:

$$
\begin{aligned}
& \frac{\partial N_{x}}{\partial x}+\frac{1}{R} \frac{\partial N_{x \theta}}{\partial \theta}-\rho h \frac{\partial^{2} u}{\partial t^{2}}=0 \\
& \frac{\partial S_{x}}{\partial x}-\frac{1}{R} \frac{\partial M_{x \theta}}{\partial x}+\frac{1}{R} \frac{\partial N_{\theta}}{\partial \theta}+\frac{Q_{\theta}}{R}-\rho h \frac{\partial^{2} v}{\partial t^{2}}=0 \\
& \frac{\partial V_{x}}{\partial x}-\frac{1}{R} \frac{\partial M_{x \theta}}{\partial x \partial \theta}+\frac{1}{R} \frac{\partial Q_{\theta}}{\partial \theta}-\frac{N_{\theta}}{R}-\rho h \frac{\partial^{2} w}{\partial t^{2}}=0 \\
& N_{x}=K\left[\frac{\partial u}{\partial x}+\mu\left(\frac{1}{R} \frac{\partial v}{\partial \theta}+\frac{w}{R}\right)\right] \\
& M_{x}=D\left(\frac{\partial \varphi_{x}}{\partial x}-\frac{\mu}{R^{2}} \frac{\partial^{2} w}{\partial \theta^{2}}\right) \\
& V_{x}=\frac{\partial M_{x}}{x}-\frac{2}{R^{2}} D(1-\mu) \frac{\partial^{3} w}{\partial x \partial \theta^{2}}
\end{aligned}
$$




$$
\begin{aligned}
& \left.S_{x}=K \frac{1-\mu}{2}\left(\frac{1}{R} \frac{\partial u}{\partial \theta}+\frac{\partial v}{\partial x}\right)\right]-\frac{1}{R^{2}} D(1-\mu) \frac{\partial^{2} w}{\partial x \partial \theta} \\
& \varphi_{x}=-\frac{\partial w}{\partial x}
\end{aligned}
$$

Here define $\left(u^{(1)}, v^{(1)}, w\right)$ and $\left(u^{(3)}, v^{(3)}, w\right)$ as the three displacement amplitude $x, \theta, z$ of the base assembly and constraint layer in the face:

$$
\left\{\begin{array}{l}
u^{(2)}(x, \theta)=\frac{1}{2}\left[\left(u^{(3)}-\frac{h_{3}}{2} \varphi_{x}^{(3)}\right)+\left(u^{(1)}+\frac{h_{1}}{2} \varphi_{x}^{(1)}\right)\right] \\
\varphi_{x}^{(2)}(x, \theta)=\frac{1}{h_{2}}\left[\left(u^{(3)}-\frac{h_{3}}{2} \varphi_{x}^{(3)}\right)-\left(u^{(1)}+\frac{h_{1}}{2} \varphi_{x}^{(1)}\right)\right]
\end{array}\right.
$$

For the direction $\theta$ can be written as:

$$
\left\{\begin{array}{l}
v^{(2)}(x, \theta)=\frac{1}{2}\left[\left(v^{(3)}-\frac{h_{3}}{2} \varphi_{\theta}^{(3)}\right)+\left(v^{(1)}+\frac{h_{1}}{2} \varphi_{\theta}^{(1)}\right)\right] \\
\varphi_{\theta}^{(2)}(x, \theta)=\frac{1}{h_{2}}\left[\left(v^{(3)}-\frac{h_{3}}{2} \varphi_{\theta}^{(3)}\right)-\left(v^{(1)}+\frac{h_{1}}{2} \varphi_{\theta}^{(1)}\right)\right]
\end{array}\right.
$$

where, $\varphi_{x}^{(i)}$ and $\varphi_{\theta}^{(i)}(i=1,2,3)$ are defined as the torsion angle of 1, 2, 3 cross section along $x$ direction and $\theta$ direction.

For fundamental shell and restrain shell, based on Kirchhoff plane suppose and column shell theory[7]:

$$
\varphi_{x}^{(i)}=-\frac{\partial w}{\partial x}=\varphi_{x}, \varphi_{\theta}^{(i)}=-\frac{1}{R_{i}} \frac{\partial w}{\partial \theta}, \quad i=1,3,
$$

where, $R_{i}$ is radius of curvature of middle surface of part $i$. The arbitrary point displacement of viscoelasticity, based on first-order shear deformation theory (FSDT), can be written as:

$$
\left\{\begin{array}{l}
u(x, \theta, z)=u^{(2)}(x, \theta)+z \varphi_{x}^{(2)}(x, \theta) \\
v(x, \theta, z)=v^{(2)}(x, \theta)+z \varphi_{\theta}^{(2)}(x, \theta) \\
w(x, \theta, z)=w(x, \theta)
\end{array}\right.
$$

Combining Eqs. (7) and relationship between strain and displacement under orthogonal curvilinear coordinates, the shear strain of the viscoelastic layer can be obtained as follow:

$$
\psi_{x}=\gamma_{z x}=\varphi_{x}^{(2)} \frac{\partial w}{\partial x}=\varphi_{x}^{(2)}-\varphi_{x}, \psi_{\theta}=\gamma_{z \theta}=\varphi_{\theta}^{(2)}-\frac{v^{(2)}}{R_{2}}+\frac{1}{R_{2}} \frac{\partial w}{\partial \theta}
$$

Substitution of Eqs. (4), (5) and (6) into Eqs. (8). Based on Hooke's law the shear stress amplitude in $x, \theta$ direction of viscous layer can be written as: 


$$
\left\{\begin{aligned}
& \tau_{z x}=G_{2} \gamma_{z x}=G_{2}\left\{\frac{1}{h_{2}}\left[\left(u^{(3)}-u^{(1)}\right)-\left(h_{2}+\frac{h_{1}+h_{3}}{2}\right) \varphi_{x}\right]\right\} \\
& \tau_{z \theta}=G_{2} \gamma_{z \theta}=G_{2}\left\{\left[\frac{2 R_{3}-h_{3}}{2 h_{2} R_{3}}-\frac{2 R_{3}-h_{3}}{4 R_{2} R_{3}}\right] v^{(3)}-\left[\frac{2 R_{1}+h_{1}}{2 h_{2} R_{1}}+\frac{2 R_{1}+h_{1}}{4 R_{1} R_{2}}\right] v^{(1)}\right. \\
&\left.+\left[\frac{h_{1}}{2 h_{2} R_{1}}+\frac{h_{3}}{2 h_{2} R_{3}}-\frac{h_{3}}{4 R_{2} R_{1}}+\frac{h_{1}}{4 R_{2} R_{1}}+\frac{1}{R_{2}}\right] \frac{\partial w}{\partial \theta}\right\}
\end{aligned}\right.
$$

Substitution of Eqs. (9) into differential equation of fundamental shell and state vector of restraint. The first-order differential equation can be written as[3,4]:

$$
\frac{d \mathbf{Z}(x)}{d x}=\mathbf{U Z}(x)
$$

where, $\mathbf{Z}(x)=\left[\begin{array}{llllllllllll}u^{(1)} & v^{(1)} & w & \varphi_{x} & N_{x}^{(1)} & S^{(1)} & V_{x}^{\prime} & M_{x} & u^{(3)} & v^{(3)} & N_{x}^{(3)} & S_{x}^{(3)}\end{array}\right]^{T}, V_{x}^{\prime}=V_{x}^{(1)}+V_{x}^{(3)}$

$\mathbf{U}$ is a matrix of $12 \times 12$.

Considering a solution to Eq. (10) of the form:

$$
\tilde{\mathbf{Z}}\left(L_{k}\right)=\tilde{\mathbf{G}}\left(L_{k}\right) \tilde{\mathbf{Z}}\left(L_{k-1}\right)
$$

where $L_{k}$ is the length of element $k, \mathbf{G}$ is transition matrix. The transition matrix $\mathbf{G}$ can be expression as:

$$
\tilde{\mathbf{G}}\left(L_{k}\right)=\exp \left(\tilde{\mathbf{U}} L_{k}\right)
$$

Substitution of Eqs. (12) into (11):

$$
\tilde{\mathbf{Z}}(H)=\tilde{\mathbf{T}}(\omega) \tilde{\mathbf{Z}}(0)
$$

where, $\tilde{\mathbf{T}}(\omega)=\tilde{\mathbf{G}}_{N}\left(L_{N}\right) \tilde{\mathbf{G}}_{N-1}\left(L_{N-1}\right) \cdots \tilde{\mathbf{G}}_{2}\left(L_{2}\right) \tilde{\mathbf{G}}_{1}\left(L_{1}\right)$

Considering different boundary conditions, the expression of the natural frequency and dissipation factor can be expressed as follow:

(1) Simply supported of both edge

When $x=0, v^{(1)}=w=N_{x}^{(1)}=M_{x}^{(1)}=v^{(3)}=N_{x}^{(3)}$

When $x=L, v^{(1)}=w=N_{x}^{(1)}=M_{x}^{(1)}=v^{(3)}=N_{x}^{(3)}$

$$
\left[\begin{array}{l}
0 \\
0 \\
0 \\
0 \\
0 \\
0
\end{array}\right]=\left[\begin{array}{llllll}
T_{0201} & T_{0204} & T_{0206} & T_{0207} & T_{0209} & T_{0212} \\
T_{0301} & T_{0304} & T_{0306} & T_{0307} & T_{0309} & T_{0312} \\
T_{0501} & T_{0504} & T_{0506} & T_{0507} & T_{0509} & T_{0512} \\
T_{0801} & T_{0804} & T_{0806} & T_{0807} & T_{0809} & T_{0812} \\
T_{1001} & T_{1004} & T_{1006} & T_{1007} & T_{1009} & T_{1012} \\
T_{1101} & T_{1104} & T_{1106} & T_{1107} & T_{1109} & T_{1112}
\end{array}\right]\left[\begin{array}{c}
u^{(1)} \\
\varphi_{x}^{(1)} \\
S_{x}^{(1)} \\
V_{x}^{(4)} \\
u^{(3)} \\
S_{x}^{(3)}
\end{array}\right]=\mathbf{T}^{\prime}\left[\begin{array}{c}
u^{(1)} \\
\varphi_{x}^{(1)} \\
S_{x}^{(1)} \\
V_{x}^{(4)} \\
u^{(3)} \\
S_{x}^{(3)}
\end{array}\right]
$$

where $T_{i j}(i=1,2 \cdots 12 ; j=1,2 \cdots 12)$ is coefficient of transition matrix. 
(2) Clamped supported of both edges

When $x=0, u^{(1)}=v^{(1)}=w=\varphi_{x}=u^{(3)}=v^{(3)}=0$

When $x=L, u^{(1)}=v^{(1)}=w=\varphi_{x}=u^{(3)}=v^{(3)}=0$

$$
\mathbf{T}^{\prime}=\left[\begin{array}{llllll}
T_{0105} & T_{0106} & T_{0107} & T_{0108} & T_{0111} & T_{0112} \\
T_{0205} & T_{0206} & T_{0207} & T_{0208} & T_{0211} & T_{0212} \\
T_{0305} & T_{0306} & T_{0307} & T_{0308} & T_{0311} & T_{0312} \\
T_{0405} & T_{0406} & T_{0407} & T_{0408} & T_{0411} & T_{0412} \\
T_{0905} & T_{0906} & T_{0907} & T_{0908} & T_{0911} & T_{0912} \\
T_{1005} & T_{1006} & T_{1007} & T_{1008} & T_{1011} & T_{1012}
\end{array}\right] .
$$

Other different boundary conditions can be solved as above.

The condition of solutions for Eq. (13) leads to a characteristic equation:

$$
\operatorname{det}\left(\mathbf{T}^{\prime}\right)=0
$$

From which the complex number eigenvalue $s_{i}$. The natural frequency of CLD shell is given as follow:

$$
f=\operatorname{Re}\left(s_{i}\right) / 2 \pi .
$$

The dissipation factor of the CLD shell can be expressed as follow:

$$
\eta=2 \operatorname{Im}\left(s_{i}\right) / \sqrt{\left(\operatorname{Re}\left(s_{i}\right)\right)^{2}+\left(\operatorname{Im}\left(s_{i}\right)\right)^{2}} .
$$

\section{Simulation result and discussions}

Geometric and material parameters of the CLD shell as follow:

$L=100 \mathrm{~m}, h_{1}=2 \mathrm{~mm}, h_{2}=0.5 \mathrm{~mm}, h_{3}=0.5 \mathrm{~m}$, $R_{1}=143 \mathrm{~mm}, \quad E_{1}=212 \mathrm{Gpa}, \quad E_{3}=70 \mathrm{Gpa}$ $\mu_{1}=0.3, \quad \mu_{3}=0.3, \quad \rho_{1}=7850 \mathrm{~kg} / \mathrm{m}^{3}$, $\rho_{3}=2700 \mathrm{~kg} / \mathrm{m}^{3} \quad, \quad \rho_{2}=999 \mathrm{~kg} / \mathrm{m}^{3}$, $G_{2}=0.896(1+0.9683 \mathrm{i}) \mathrm{Mpa}$.

The shell is divided into 8 sections and the length of each part is $12.5 \mathrm{~mm}$. The natural frequency of different boundary conditions are calculated. The result is shown as Fig. 1,2 and Fig. 3 shows the dissipation factor of different boundary conditions. The results show that the

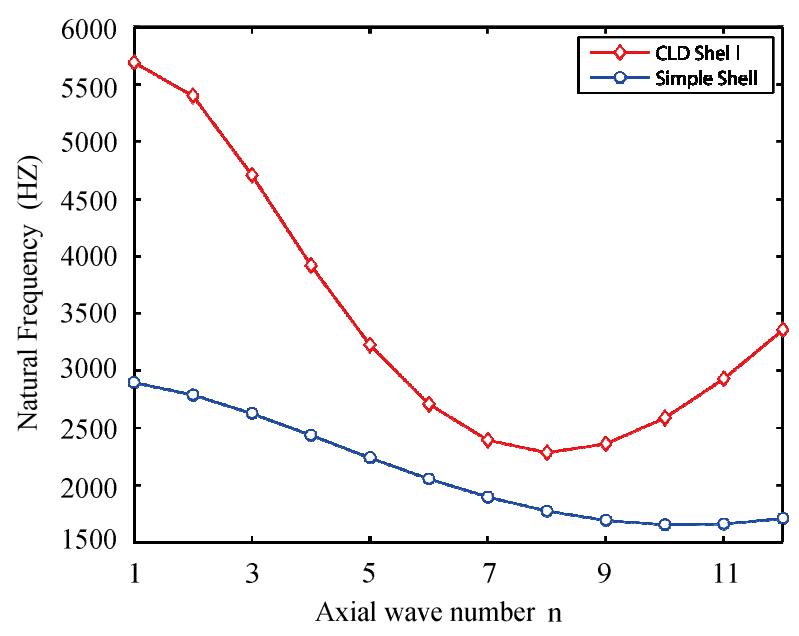

Fig. 2 Nature frequency under s-s boundary conditions CLD shell's natural frequency is increased because of the damping. 


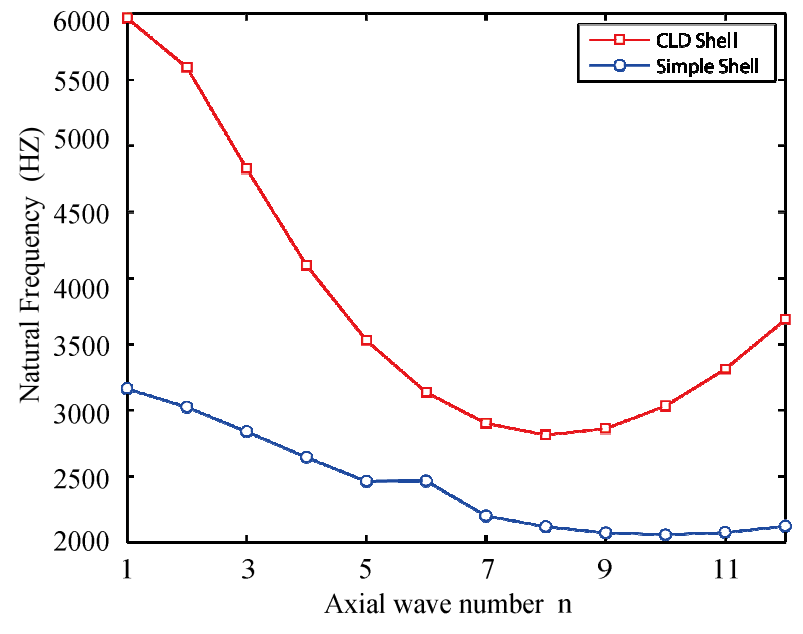

Fig. 3 Nature frequency of clamped supported of both edges

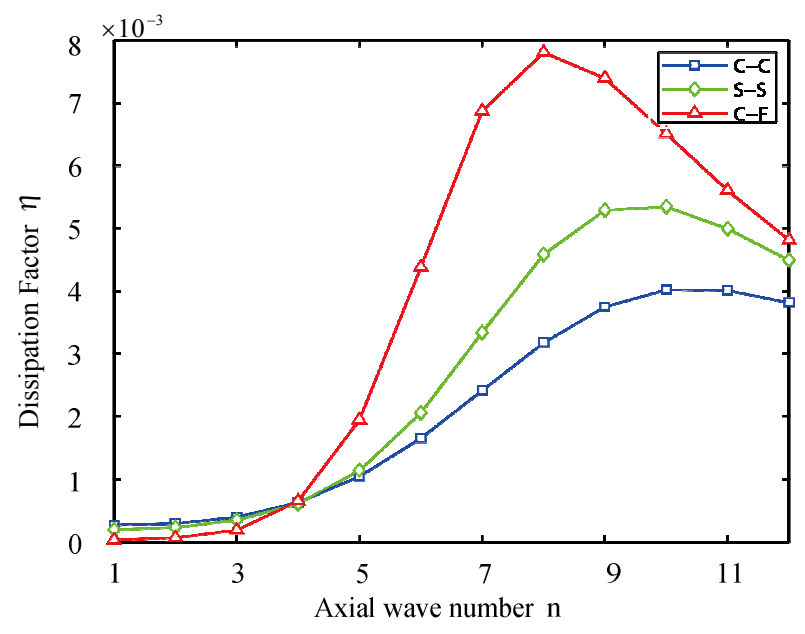

Fig. 4 Loss factor under different boundary conditions

\section{Conclusion}

The free vibration characters of the CLD shell is researched in this paper. The transfer matrix method is introduced to get the entirety transfer matrix and the expression of different frequency and dissipation factor. Comparing two typical boundary conditions, the influence of damping to vibration characteristics is conclusion as follow.

(1) The model of transfer matrix method is simply and easy to solve by computer program compared with analytical method.

(2) The influence of damping is significant as the result shown, in this case the CLD shell has higher natural frequency than traditional shell.

\section{Acknowledgements}

This work was financially supported by the Fundamental Research Funds for the Central Universities (N100603009, N100503001).

\section{Reference}

[1] R.H. Liu, Plate and shell analysis and the application, Engineering Sciences. 11 (2000) 60-67.

[2] Y. Zhou, Viscoelastic damping suspension structure design, Wuhan University of Technology Press, Wuhan, 2006.

[3] E.Q. Li, Daokui, Li, Guojin Tang, Yongjun Lei, Dynamic analysis of Constraint Layer Damping Cylinder Shell, Engineering Mechanics. 25 (2008) 6-11.

[4] Y. Xiang, Y.Y. Huang, Passive constraint layer damping vibration damping cylinder shell and the analysis of a new matrix method, Applied Mathematics and Mechanics. 29 (2008) 1443-1456.

[5] Z.Y. Cao, Plate and shell vibration theory, China Railway Press, Beijing, 1989.

[6] Q. Han, X.Q Huang, Higher plate and shell theory, Science Press, Beijing, 2002.

[7] Q.K. Han, W. Sun, Elasticity and Finite Element Method, Northeastern university Press, Shenyang, 2009. 